\title{
Combined amino acid PET-MRI for identifying recurrence in post-treatment gliomas: together we grow
}

Shumyla Jabeen ${ }^{1}$, Arpana Arbind ${ }^{2}$, Dinesh Kumarr ${ }^{2}$, Pardeep Kumar Singh ${ }^{2}$, Jitender Saini ${ }^{2}$, Nishanth Sadashiva ${ }^{3}$, Uday Krishna ${ }^{4}$, Arivazhagan Arimappamagan ${ }^{3}$, Vani Santosh ${ }^{5}$ and Chandana Nagaraj ${ }^{2^{*}}$ (i)

\author{
* Correspondence: dr.chandana@ \\ outlook.com \\ ${ }^{2}$ Department of Neuroimaging and \\ Interventional Radiology, National \\ Institute of Mental Health and \\ Neurosciences, Bengaluru, Karnataka \\ 560029, India \\ Full list of author information is \\ available at the end of the article
}

Abstract: Purpose: The aim of this study is to compare the diagnostic accuracy of amino acid PET, MR perfusion and diffusion as stand-alone modalities and in combination in identifying recurrence in post-treatment gliomas and to qualitatively assess spatial concordance between the three modalities using simultaneous PET-MR acquisition.

Methods: A retrospective review of 48 cases of post-treatment gliomas who underwent simultaneous PET-MRI using C11 methionine as radiotracer was performed. MR perfusion and diffusion sequences were acquired during the PET study. The following parameters were obtained: $T B R_{\text {max }}, T B R_{\text {mean }}, S U V_{\text {max }}$, and $S U V_{\text {mean }}$ from the PET images; rCBV from perfusion; and $A D C_{\text {mean }}$ and $A D C_{\text {ratio }}$ from the diffusion images. The final diagnosis was based on clinical/imaging follow-up and histopathology when available. ROC curve analysis in combination with logistic regression analysis was used to compare the diagnostic performance. Spatial concordance between modalities was graded as 0,1 , and 2 representing discordance, $<50 \%$ and $>50 \%$ concordance respectively.

Results: There were 35 cases of recurrence and 13 cases of post-treatment changes without recurrence. The highest area under curve (AUC) was obtained for TBR $\max$ followed by rCBV and $A D C_{\text {ratio. }}$. The AUC increased significantly with a combination

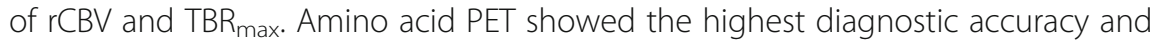
maximum agreement with the final diagnosis. There was discordance between ADC and PET in 22.9\%, between rCBV and PET in 16.7\% and between PET and contrast enhancement in $14.6 \%$ cases.

Conclusion: Amino acid PET had the highest diagnostic accuracy in identifying recurrence in post-treatment gliomas. Combination of PET with MRI further increased the AUC thus improving the diagnostic performance.

Keywords: Amino acid PET, Gliomas, Radiation necrosis, Recurrence, Perfusion, Diffusion (c) The Author(s). 2021 Open Access This article is licensed under a Creative Commons Attribution 4.0 International License, which permits use, sharing, adaptation, distribution and reproduction in any medium or format, as long as you give appropriate credit to the original author(s) and the source, provide a link to the Creative Commons licence, and indicate if changes were made. The images or other third party material in this article are included in the article's Creative Commons licence, unless indicated otherwise in a credit line to the material. If material is not included in the article's Creative Commons licence and your intended use is not permitted by statutory regulation or exceeds the permitted use, you will need to obtain permission directly from the copyright holder. To view a copy of this licence, visit http://creativecommons.org/licenses/by/4.0/. 


\section{Introduction}

Gliomas are the most common primary brain neoplasms (Ostrom et al., 2018). Maximal safe surgical excision with adjuvant chemoradiotherapy is the mainstay of treatment for high grade gliomas (grade III and IV). However, recurrence rates continue to remain high with poor overall survival despite treatment especially in case of glioblastoma (Weathers \& Gilbert, 2015; Stupp et al., 2005a). This mandates stringent post-treatment clinical and imaging surveillance. Magnetic resonance imaging (MRI) is indispensable for assessment of disease burden and response following therapy. Recent literature has brought focus on the various treatment effects in brain due to radiation therapy, chemotherapy, and immunotherapy, the imaging features of which can often resemble tumor recurrence. Distinguishing recurrence from treatment-related changes as well as identifying recurrence in a background of such changes is extremely challenging and has profound prognostic and therapeutic implications. There is also a need to set out robust criteria for enrollment in clinical trials and evaluation of efficacy of new emerging therapies. Over several years, the consensus Response Assessment in Neuro-Oncology (RANO) criteria based on conventional MRI and clinical assessment have been used toward achieving this end (Wen et al., 2017). Although, there are guidelines for ruling out pseudo-progression with these criteria, this requires a waiting period of 3 months following chemoradiotherapy during which a definitive diagnosis cannot be made (Wen et al., 2017). Besides, recurrent tumor can often co-exist with radiation necrosis (Sugahara et al, 2000) and the two cannot be resolved on conventional imaging alone. Advanced MRI techniques like perfusion, spectroscopy, and quantification of diffusion parameters have shown to be useful in detecting progressive disease as highlighted in several earlier studies. However, at the cost of various challenges in interpretation due to overlapping parameters (Seeger et al., 2013; Keunen et al., 2014; Heiss et al., 2011). Molecular imaging, which reflects the tumoral physiologic milieu, acts as a problem-solving tool complementary to MRI. FDG-PET has an established role in post-treatment imaging of gliomas (Wang et al., 2015; Nozawa et al., 2015; Nihashi et al., 2013). However, increased uptake by inflammatory cells and high background uptake by the normal brain parenchyma can lead to a false diagnosis (Nihashi et al., 2013). In this regard, PET imaging with alternate metabolites like $\mathrm{C}-11$ methionine may be advantageous in view of reduced normal parenchymal uptake. Also, discordance between MR perfusion and FDG-PET was demonstrated in an earlier study highlighting the different functional parameters which they reflect (Jena et al., 2017). Besides, in most of the early studies based on advanced MRI and PET imaging, the two studies were performed at different time points thus adding to the complexity of the results. In this study, we compared the diagnostic accuracies of amino acid PET, MR perfusion, and diffusion as standalone modalities and in combination in identifying recurrence in treated gliomas using simultaneous PET-MR acquisition. In addition, a qualitative assessment of spatial concordance between increased metabolic uptake on PET, elevated perfusion on dynamic susceptibility contrast (DSC) MRI, and restricted diffusion on diffusion-weighted imaging was performed. 


\section{Material and methods \\ Type of study}

This single institute retrospective study was carried out at a dedicated quaternary care center providing neurosurgical and neuro-imaging services for patients with brain tumors. Written informed consent was obtained from all subjects prior to imaging. The study was approved by institutional ethics committee review board.

\section{Subjects}

All cases of histopathologically proven glial tumors who had undergone surgical resection followed by fractionated radiotherapy with or without standard chemotherapy with temozolomide who underwent simultaneous amino acid PET-MRI with suspected recurrence between January 2019 and March 2020 were included in the study. Exclusion criteria included non-glial primary brain tumors, metastatic lesions, and standard contraindications for PET and/or MRI like pregnancy, end-stage renal disease, and presence of a cardiac pacemaker or MRI incompatible metallic implants. Molecular biomarker data were obtained from histopathology reports of the primary tumor at initial biopsy or resection. Radiation therapy to a total dose of 60 Gy in 30 fractions was usually initiated within 6 weeks after surgery. Temozolomide was given concurrently and sequentially, per Stupp et al. (Stupp et al., 2005b).

\section{Imaging technique}

All patients underwent simultaneous amino-acid PET-MR imaging on a 3 Tesla SIEMENS, Biograph mMR scanner (Erlangen, Germany). All patients were fasted 4-6 h prior to scanning for baseline stable metabolic conditions. On the day of imaging, all patients were injected 360-378 MBq of C11 methionine on the table through IV cannula. Simultaneous acquisition of PET images was performed along with UTE MR attenuation correction sequence (MRAC) along with other standard and advanced MRI sequences for $40 \mathrm{~min}$ in LIST MODE. PET images were acquired using the following parameters: $500 \mathrm{~mm}$ FOV, $400 \mathrm{~mm}$ anterior-posterior FOV, 1.0 zoom, 3 interactions, 21 subsets, HD PET reconstruction method, and $2.0 \mathrm{~mm}$ Gaussian filter.

The following MR sequences were obtained during the PET acquisition: 3D fluidattenuated inversion recovery (FLAIR)- TR/TE $=5000 / 385 \mathrm{~ms}$, TI $=1800 \mathrm{~ms}$, voxel size $=1 \times 1 \times 1 \mathrm{~mm}$, FOV $=256 \times 256$; axial T1 spin echo-TR $/ \mathrm{TE}=550 / 15 \mathrm{~ms}$, slice thickness $-4 \mathrm{~mm}, \mathrm{FOV}=230 \times 230$; axial T2 spin echo-TR/TE $=5500 / 92 \mathrm{~ms}$, slice thickness-4 mm, FOV = $230 \times 230$; axial susceptibility-weighted imaging (SWI)-TR/TE $=27 / 20 \mathrm{~ms}$, flip angle $=15^{\circ}$, slice thickness $=2 \mathrm{~mm}$, FOV $=230 \times 230$; axial diffusionweighted imaging (DWI)-TR/TE $=3900 / 81 \mathrm{~ms}$, slice thickness $-4 \mathrm{~mm}, \mathrm{FOV}=230 \times$ 230 at $b$ values of 50 and 1000 .

DSC perfusion was performed after the administration of gadolinium-based contrast agent in a dose of $0.1-0.15 \mathrm{mmol} / \mathrm{kg}$ body weight at a rate of $5-6 \mathrm{ml} / \mathrm{s}$ using a dual chamber injector connected to a 16-gauge cannula placed in the antecubital vein followed by $25 \mathrm{ml}$ saline chase at the same rate. Echo planar sequence was acquired with parameters as follows $\mathrm{TR} / \mathrm{TE}=1900 / 30 \mathrm{~ms}$, flip angle $=90^{\circ}$, slice thickness $=4$ $\mathrm{mm}$, FOV $=230 \times 230$, no. of slices $=25$. This was followed by acquisition of post contrast T1 magnetization prepared rapid gradient-echo (MPRAGE) sequence with 
$\mathrm{TR} / \mathrm{TE}=2200 / 2.33 \mathrm{~ms}, \mathrm{TI}=900 \mathrm{~ms}$, flip angle $=8^{\circ}, \mathrm{FOV}=256 \times 256$, voxel size $=1 \times$ $1 \times 1 \mathrm{~mm}$.

\section{Image analysis}

The PET and MRI scans were analyzed by a nuclear medicine specialist and neuroradiologist respectively.

\section{PET analysis}

\section{Quantitative ROI analysis}

C11 methionine PET images were loaded into SIEMENS SYNGO Via (VB30) workstation after correcting for partial volume effects (PVE) using Siemens E7 tools (Fig. 1A, B). The 3D ROI was drawn semi-automatically using an individually adapted isocontour of the tumor maximum using a standard ROI with a fixed diameter of $1.6 \mathrm{~cm}$ centered on the tumor maximum yielding a volume of $2 \mathrm{ml}$ (Fig. 1C). Similar mirror ROI was placed in the contralateral brain parenchyma to calculate the background /normal brain parenchymal uptake (Fig. 1C). The values SUV max and SUV mean were obtained for both tumor and normal brain parenchyma and tabulated. Ratio TBR max and TBR mean (tumor to normal brain/background) were calculated for statistical analysis.

\section{MRI analysis}

\section{Quantitative ROI analysis}

DSC perfusion, diffusion trace images with ADC maps, and post-contrast images were loaded into Philips Intellispace Portal version 6.0. Perfusion images were processed using the leakage correction algorithm. The colored CBV maps were co-registered with the post-contrast image. After visual assessment, three to four ROIs were drawn in the areas showing elevated perfusion and the ROI with the maximum value was used for further analysis. A mirror ROI was placed in the contralateral normal white matter and the relative $\mathrm{CBV}$ ratio obtained. The ADC maps were also co-registered with the postcontrast images and ROI drawn in the same region to obtain the mean ADC value. Another ROI was drawn in the contralateral normal white matter and the ADC ratio calculated.

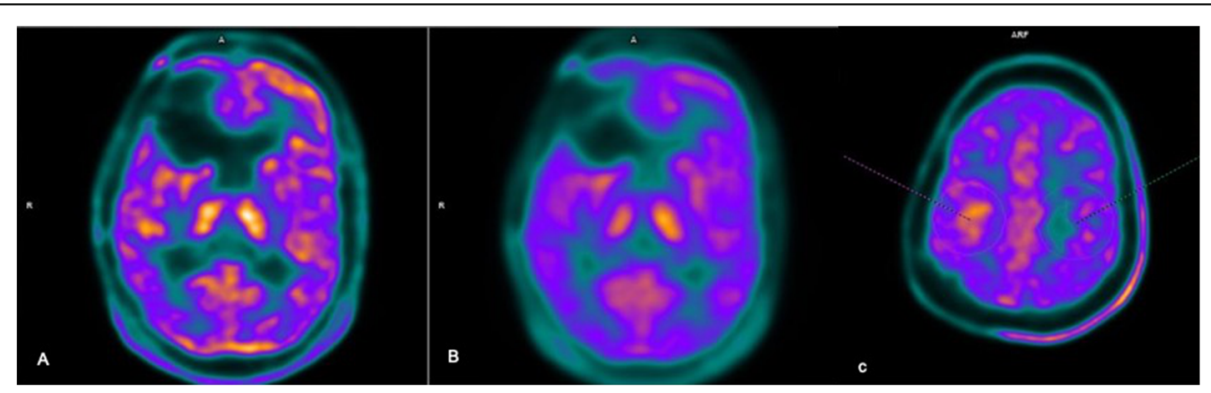

Fig. 1 LIST mode UTE MRAC sequence reconstructed PET images (A) and images reprocessed on E7 tools SIEMENS for correction of partial volume effects (PVE) (B). 3D ROI was drawn semi-automatically using an individually adapted isocontour of the tumor maximum using a standard ROI with a fixed diameter of 1.6 $\mathrm{cm}$ centered on the tumor maximum yielding a volume of $2 \mathrm{ml}(\mathbf{C})$ 


\section{Qualitative grading of diffusion restriction}

A qualitative ordinal scale was used to grade the degree of diffusion restriction on ADC maps with grade 1 assigned when the region of interest appeared brighter than the normal white matter, grade 2 when the signal intensity is same as white matter, grade 3 less than white matter, and grade 4 avid, unequivocal diffusion restriction.

\section{Qualitative visual assessment for detecting recurrence}

A visual analysis of the rCBV maps, ADC maps, and post-contrast images was independently performed to assess for the presence of recurrence without quantification.

\section{Spatial concordance between PET, perfusion, and diffusion}

The colored rCBV map, ADC map, and post-contrast image each were independently compared to the PET image and the spatial concordance between the area of uptake, elevated rCBV on perfusion, restricted diffusion on ADC map, and enhancement on post-contrast image graded as follows: grade 0-discordance, 1-fair (less than 50\%), and 2-moderate (more than 50\%) concordance between area of PET uptake and elevated perfusion on $\mathrm{rCBV}$ map/restriction on $\mathrm{ADC}$ map/enhancement on post-contrast $\mathrm{T} 1$ MPRAGE.

\section{Final diagnosis}

The final diagnosis of recurrence was based on histopathology when available and on clinical and/or imaging follow up for cases where it was not. Disease progression clinically or on imaging was classified as recurrence.

\section{Statistical analysis}

Quantitative variables were expressed as median or mean with standard deviation. Qualitative variables were expressed as percentages. The PET, perfusion, and diffusion parameters between the two groups were compared using the Mann-Whitney $U$ test. Receiver operating characteristic (ROC) curve analysis was used to assess the diagnostic performance of each parameter in detecting recurrence. ROC curve analysis in combination with logistic regression analysis was used to measure the area under curve of various combination of parameters. The degree of agreement between perfusion, diffusion, contrast enhancement, PET, and final diagnosis was estimated using the Cohen kappa statistic with values of .01-.20, .21-.40, .41-.60, .61-.80, and .81-1.00 indicating slight, fair, moderate, substantial, and perfect agreement. Spatial concordance between rCBV maps, ADC maps, post-contrast MRI, and PET was expressed as percentages. All analysis was performed on IBM SPSS version 26. A $p$ value of less than 0.05 was regarded as significant.

\section{Results}

Demographics, primary lesion characteristics, and final diagnosis

There were 48 cases of post-treatment glial brain tumors who underwent simultaneous PET-MR imaging during the study period. The mean age at diagnosis was $39.9 \pm 12.5$ years with range of $8-71$ years and median 39.5 years. There were 31 males and 17 females with a male:female ratio of approximately 1.8:1. Patient demographics, tumor 
characteristics, treatment details, duration following treatment when recurrence was suspected, and follow-up duration after imaging are detailed in Table 1. Post C11 methionine imaging the final diagnosis was considered as recurrence in 35 cases out of which 9 were confirmed on histopathology and 26 had clinical/imaging evidence of progressive disease. Thirteen cases had no evidence of recurrence on clinical/imaging follow-up. The median duration of follow-up after imaging was 7 months ranging between 1 and 14 months (mean $7.5 \pm 4$ months).

Comparison of quantitative PET, perfusion, and diffusion parameters between recurrence and radiation necrosis

The mean value of each parameter is shown in Table 2. Intergroup comparison using the Mann-Whitney $U$ test showed a significant difference between the two groups for all PET, perfusion, and diffusion parameters. $\mathrm{SUV}_{\max }, \mathrm{TBR}_{\max }$, and $\mathrm{TBR}_{\text {mean }}$ reached the highest level of significance ( $p$ value $<0.001)$ followed by rCBV $(p$ value $=.001)$.

\section{Diagnostic performance of PET and MRI parameters}

The ROC curves for each parameter are shown in Figs. 2 and 3 with values detailed in Table 3. Among the PET parameters, the highest area under curve (AUC) was obtained for the $\mathrm{TBR}_{\max }$ followed by $\mathrm{SUV}_{\text {max }}, \mathrm{TBR}_{\text {mean }}$, and $\mathrm{SUV}_{\text {mean }}$ in that order. For MRI, rCBV ratio showed the highest area under curve followed by ADC ratio, qualitative diffusion restriction grade, and mean ADC.

The sensitivity, specificity, positive predictive value, negative predictive value, and diagnostic accuracy for each parameter using an appropriate threshold value determined from the coordinates of the ROC curve are shown in Table 3.

A combined ROC curve analysis was performed with $\mathrm{rCBV}$ ratio in combination with $\mathrm{TBR}_{\max }$ and $\mathrm{rCBV}$ along with $\mathrm{TBR}_{\max }$ and $\mathrm{ADC}$ ratio. The $\mathrm{AUC}$ for $\mathrm{rCBV}+\mathrm{TBR}_{\max }$ was higher than that of $\mathrm{rCBV}$ alone (0.908 vs. 0.823) showing higher diagnostic accuracy which was statistically significant $(p=0.034)$. Adding ADC ratio further increased the AUC to 0.913; however, the difference was not statistically significant (Fig. 4).

Table 1 Median, mean, standard deviation (SD) for PET, perfusion, and diffusion parameters in either group with $p$ values obtained using the Mann-Whitney test

\begin{tabular}{llll}
\hline Parameters & $\begin{array}{l}\text { Recurrence }=\mathbf{3 5} \\
\text { Mean, Median (SD) }\end{array}$ & $\begin{array}{l}\text { Radiation necrosis = 13 } \\
\text { Mean, Median (SD) }\end{array}$ & $p$ value \\
\hline TBR $_{\text {max }}$ & $1.83,1.59(1.05)$ & $1.03,1.02(0.18)$ & .000 \\
TBR $_{\text {mean }}$ & $1.67,1.57(1.01)$ & $0.90,0.95(0.30)$ & .000 \\
SUV $_{\text {max }}$ & $4.72,4.36(2.15)$ & $2.66,2.64(0.80)$ & .000 \\
SUV $_{\text {mean }}$ & $2.44,2.15(1.3)$ & $1.52,1.57(0.70)$ & .019 \\
rCBV $_{\text {ratio }}$ & $2.94,2.00(1.8)$ & $0.99,0.67(0.94)$ & .001 \\
$\mathbf{A D C}_{\text {mean }}$ & $0.78,0.77(0.14)$ & $0.99,0.95(0.38)$ & .032 \\
ADC $_{\text {ratio }}$ & $0.99,0.96(0.25)$ & $1.49,1.3(0.73)$ & .004 \\
\hline
\end{tabular}

TBR tumor to background ratio, SUV standard uptake value, $r C B V$ relative cerebral blood volume, $A D C$ apparent diffusion co-efficient, $S D$ standard deviation 
Table 2 Area under curve values with sensitivity, specificity, positive predictive values (PPV), negative predictive values (NPV), and diagnostic accuracy for important PET, perfusion, and diffusion parameters

\begin{tabular}{llllllll}
\hline Parameter & AUC & $\begin{array}{l}\text { CUT- } \\
\text { OFF }\end{array}$ & $\begin{array}{l}\text { Sensitivity } \\
\text { (\%) }\end{array}$ & $\begin{array}{l}\text { Specificity } \\
\text { (\%) }\end{array}$ & $\begin{array}{l}\text { PPV } \\
\text { (\%) }\end{array}$ & $\begin{array}{l}\text { NPV } \\
\text { (\%) }\end{array}$ & $\begin{array}{l}\text { Diagnostic accuracy } \\
\text { (\%) }\end{array}$ \\
\hline TBR $_{\text {max }}$ & 0.865 & 1.23 & 81.8 & 92.3 & 91.4 & 83.5 & 87.05 \\
rCBV $_{\text {ratio }}$ & 0.823 & 1.38 & 84.8 & 76.9 & 78.6 & 83.5 & 80.85 \\
ADC $_{\text {ratio }}$ & 0.776 & 1.11 & 78.1 & 69.2 & 71.7 & 75.9 & 73.65 \\
ADC $_{\text {grade }}$ & 0.722 & $>1$ & 84 & 46.2 & 60.9 & 74.3 & 65.1 \\
\hline
\end{tabular}

$T B R$ tumor to background ratio, SUV standard uptake value, $r C B V$ relative cerebral blood volume, $A D C$ apparent diffusion co-efficient, AUC area under the curve

Agreement of PET, MR perfusion, and diffusion diagnosis on visual assessment with the final diagnosis

Using the Cohen kappa statistic, substantial agreement was seen between PET and the final diagnosis $(\mathrm{kappa}=0.766)$ with moderate agreement of rCBV maps (0.472), ADC maps (0.499), and post-contrast images (0.451).

\section{Concordance between PET, MR perfusion, and diffusion}

There was discordance between ADC and PET in 11 cases (22.9\%), between rCBV and PET in 8 cases (16.7\%), and between PET and contrast enhancement in 7 (14.6\%) cases (Fig. 5). In the case of 11 cases with diffusion-PET discordance, PET correctly classified 9 out of 11 cases as recurrence (Fig. 6). Out of 8 cases of discordance between perfusion and PET, only one was incorrectly classified as recurrence on PET while in 7 cases of PET-contrast enhancement discordance, one case was misclassified on PET as recurrence. All instances of incorrect classification on PET were a result of false positive diagnosis in which radiation necrosis was diagnosed as recurrence (Fig. 7). Area of PET uptake and elevated perfusion on MRI showed partial (less than 50\%) and near complete (more than 50\%) concordance in 12.5 and $70.9 \%$ cases respectively. On ADC maps, partial and near complete concordance of area of diffusion restriction with PET

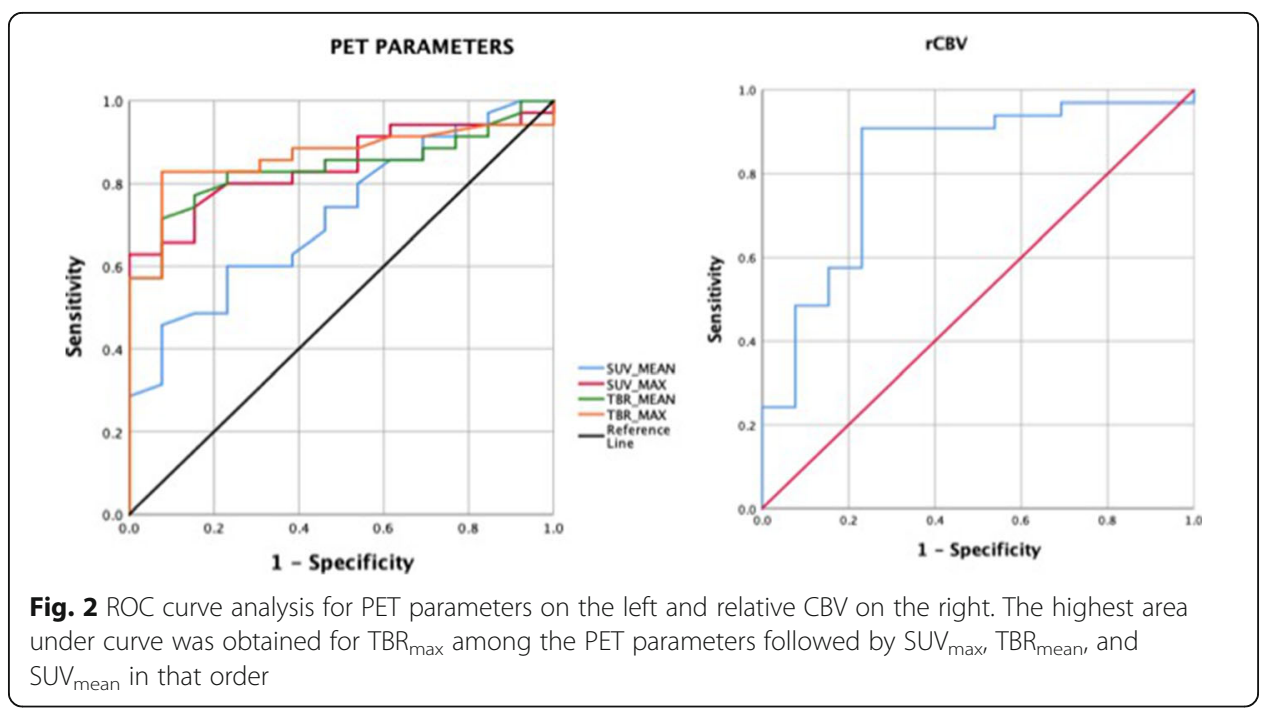




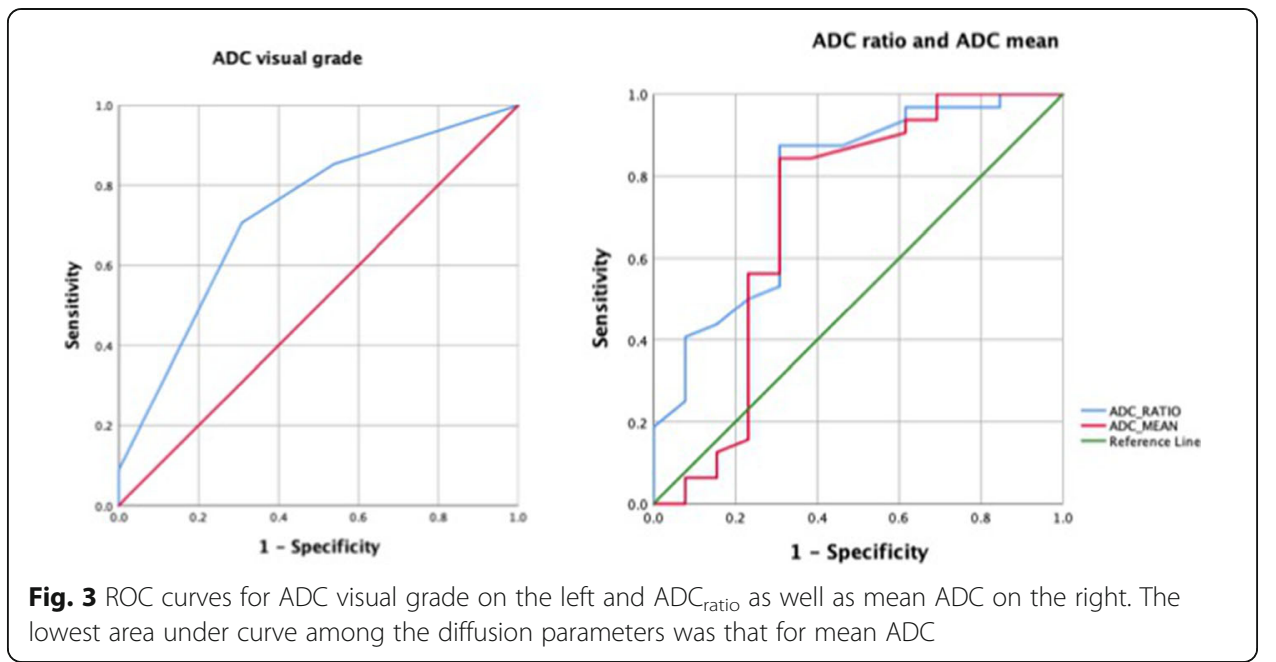

uptake was seen in 14.6 and $62.5 \%$ cases respectively. There was $16.7 \%$ partial and 68.8\% near complete concordance between contrast enhancement and PET uptake.

\section{Discussion}

Differentiating recurrence from post-treatment changes in gliomas is critical for patient management. The former requires re-exploration and resection of the tumor, while the latter may be managed medically with steroids. Besides radiation necrosis, other treatment-related changes like pseudo-progression and pseudo-response add to the diagnostic confusion. Radiation necrosis occurs around 3 to 12 months following radiotherapy and presents as an increase in post-contrast enhancement on conventional MRI (Verma et al., 2013). Pseudoprogression results from exaggerated response to treatment and is generally seen within 3 months following radiotherapy with or without chemotherapy. Pseudoresponse occurs due to reduced vascularity following treatment with the anti-angiogenic drug bevacizumab resulting in a decrease in enhancement in an otherwise viable tumor (Zikou et al., 2018).

Conventional magnetic resonance imaging, commonly used for post-treatment follow up of gliomas, falters in differentiating recurrence from treatment related changes since both recurrences, early and delayed radiation changes show an increase in enhancement on post-contrast images secondary to disruption of the blood brain barrier (Verma et al., 2013; van Dijken et al., 2019). MR perfusion, diffusion, and molecular imaging techniques provide surrogate markers for angiogenesis and cell proliferation which are features of recurrent tumor (Ronca et al., 2017). Several studies have been performed using these techniques in isolation (Wang et al., 2015; Nihashi et al., 2013; van Dijken et al., 2019; Barajas Jr et al., 2009) and combination (Seeger et al., 2013; Keunen et al., 2014; Heiss et al., 2011; Jena et al., 2017; Nael et al., 2018) with variable results.

Molecular imaging is a useful adjunct to advanced MRI in identifying recurrence in post-treatment gliomas. Most of the PET studies have been performed using 18-fluorodeoxyglucose (FDG) as the radiotracer. Although some studies reported a low specificity for FDG-PET in detecting recurrence (Ricci et al., 1998; Hustinx et al., 2005), a meta-analysis by Wang et al. (Wang et al., 2015) revealed diagnostic performance 


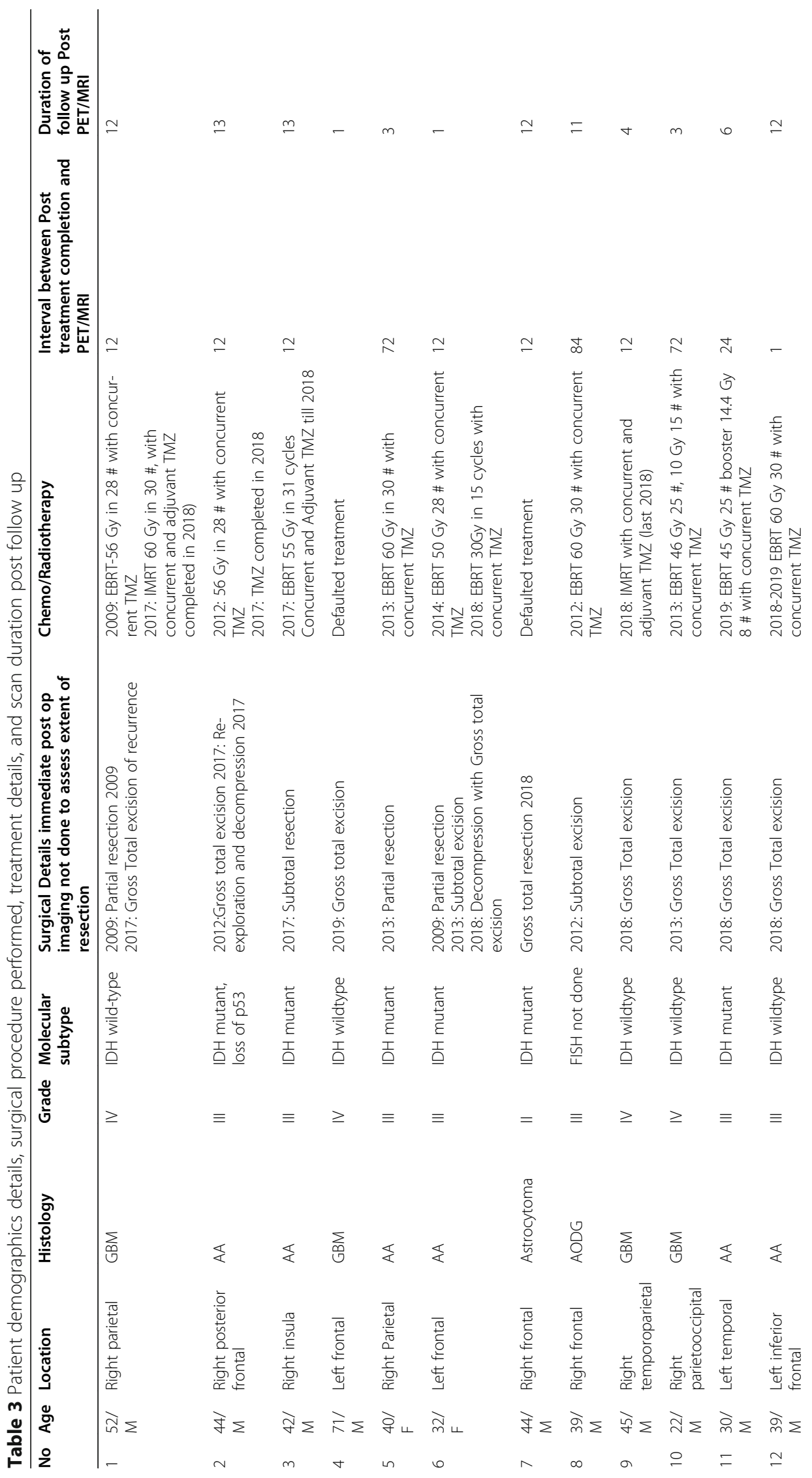




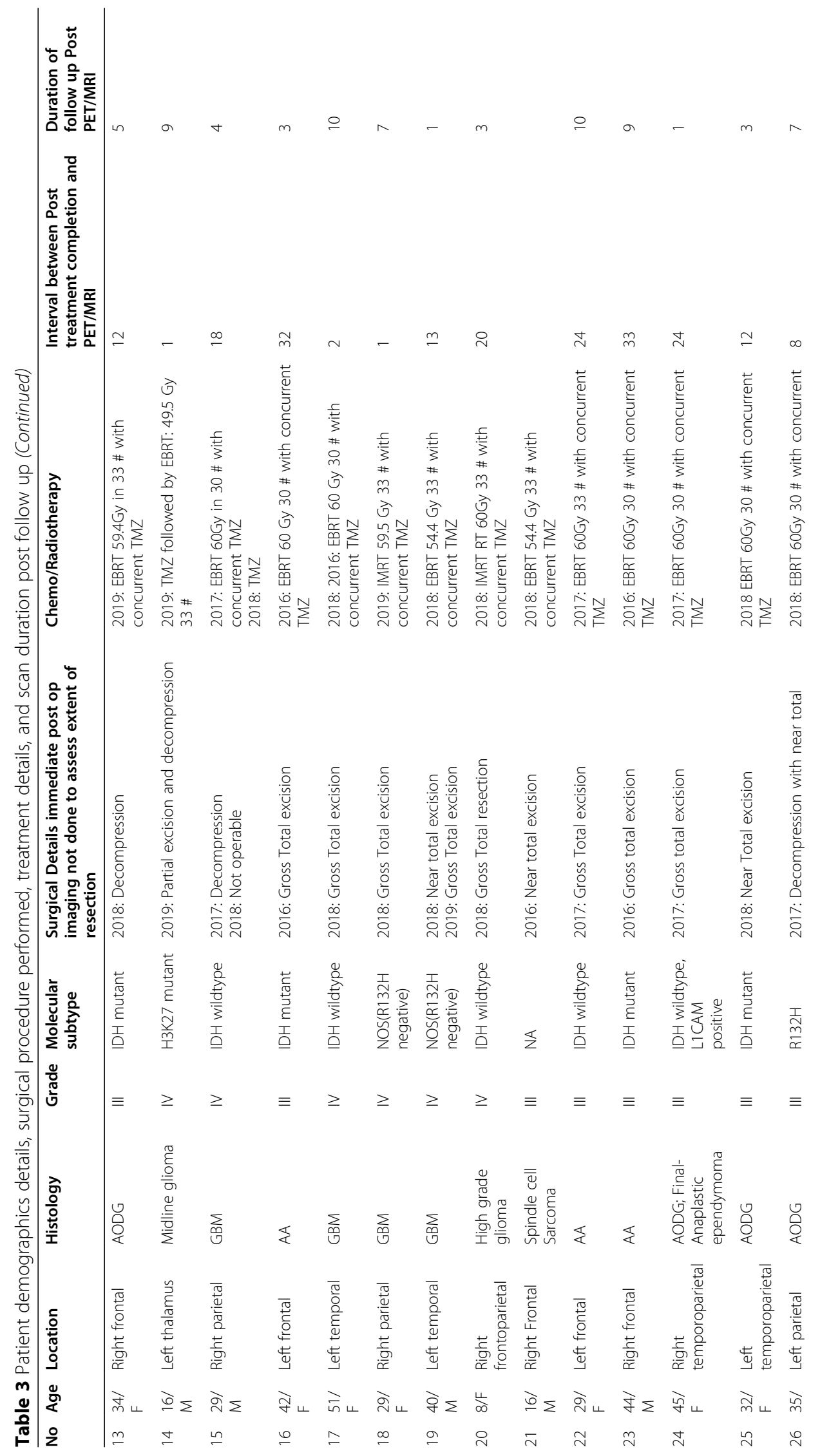




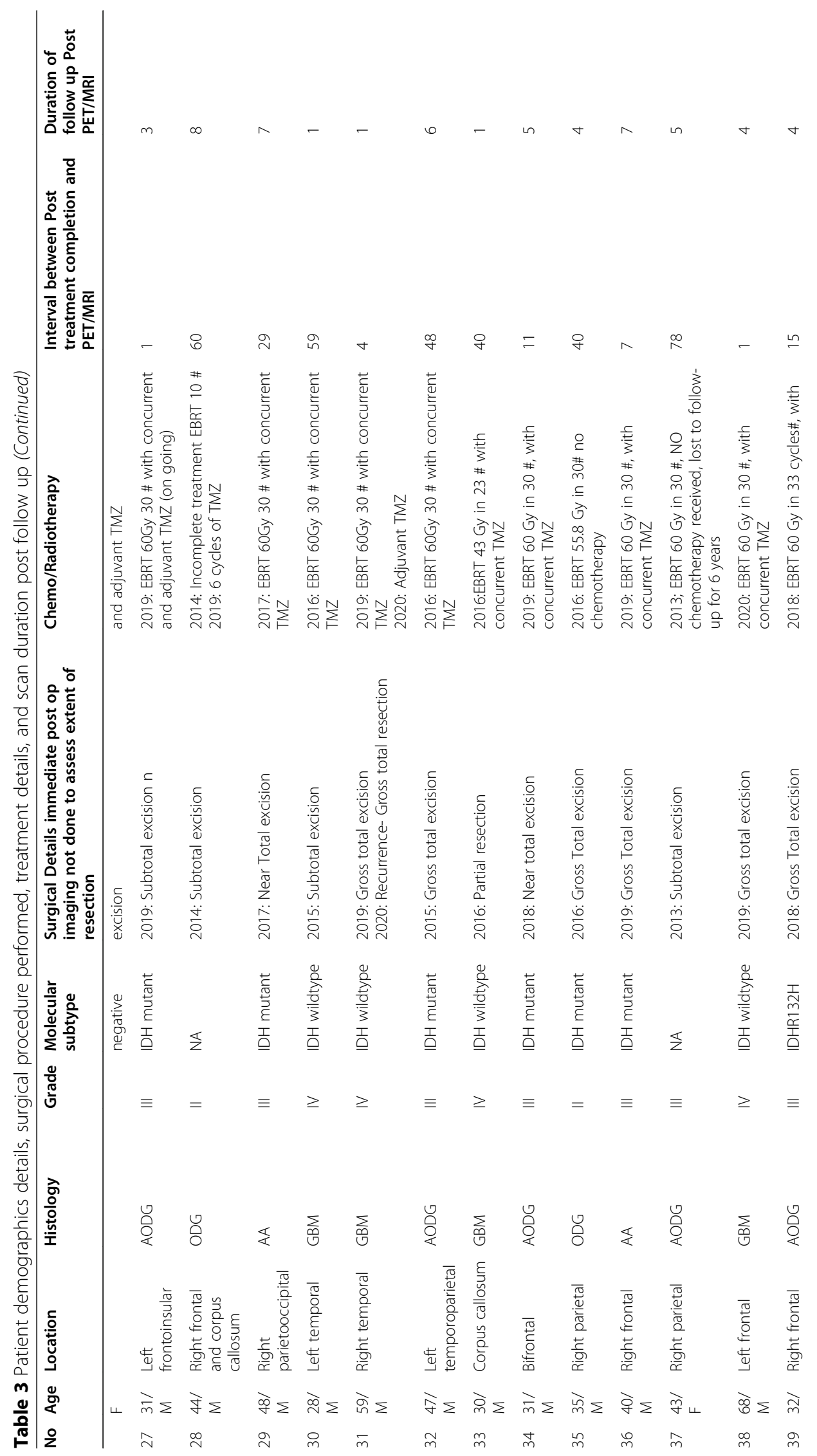




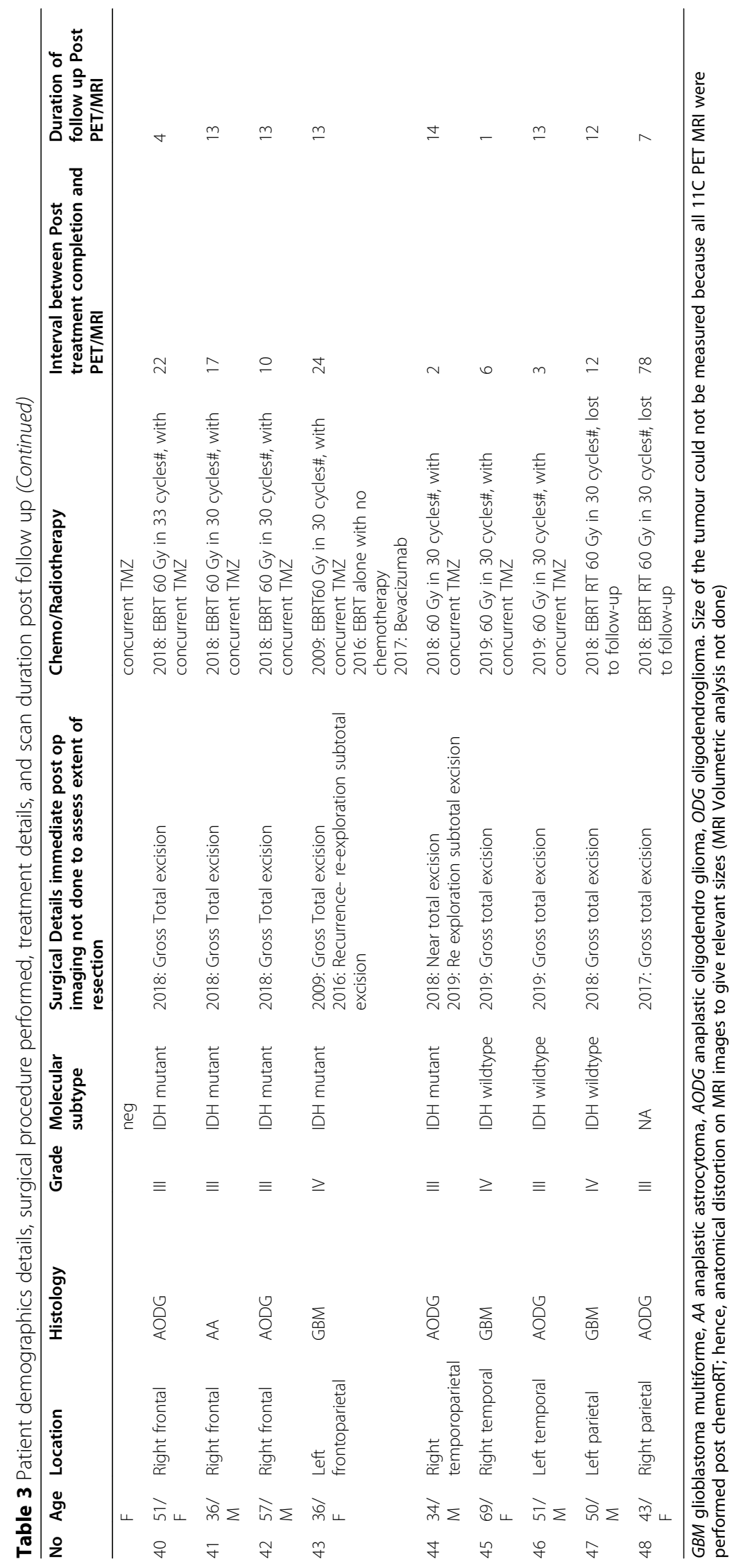




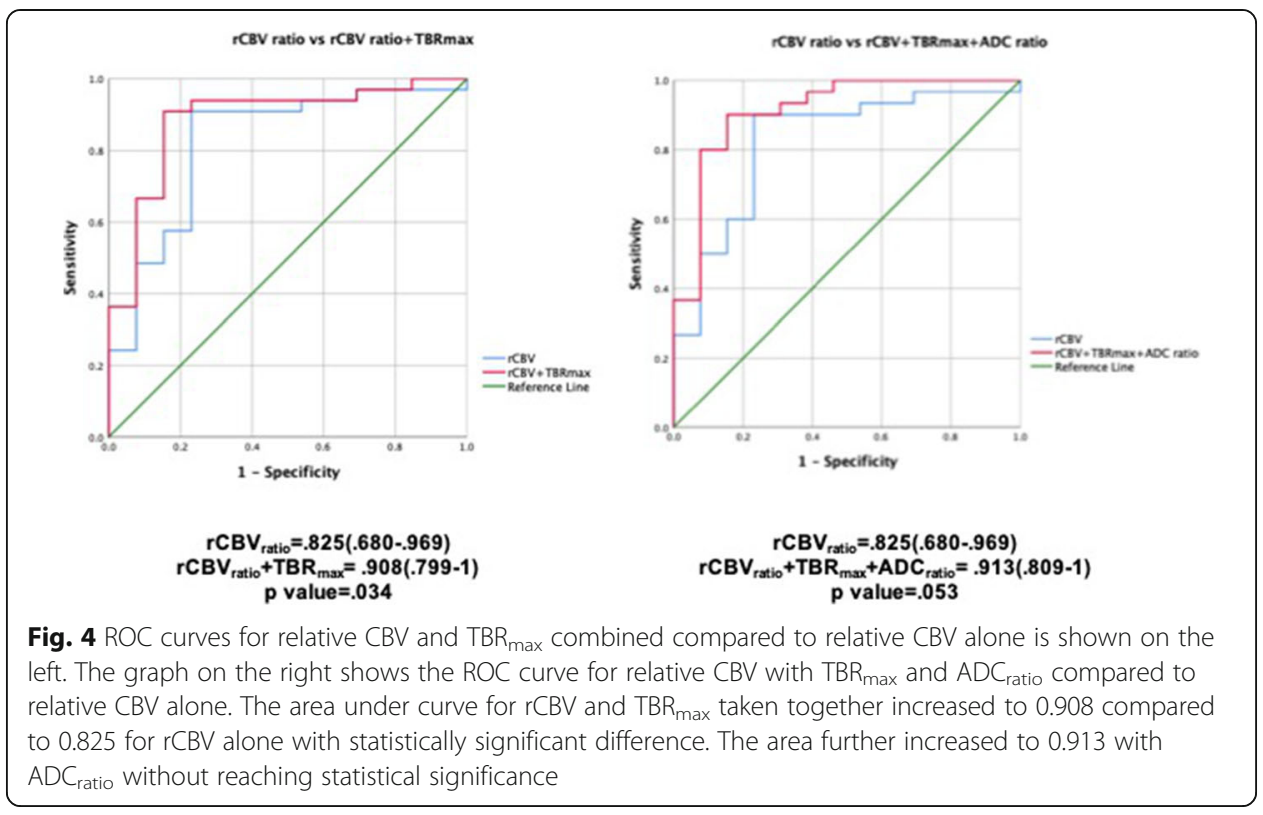

comparable to magnetic resonance spectroscopy (MRS). In this metanalysis comparing FDG-PET, C-11 methionine PET, and MRS, the pooled sensitivity for FDG-PET was the lowest at $70 \%$ and the specificity was highest at $88 \%$ (Wang et al., 2015). The heterogeneity in data related to FDG-PET and lower sensitivity may partly be attributed to the uptake of FDG by the normal brain parenchyma which leads to misdiagnosis in some cases (Soni et al., 2020). In this regard, amino acid tracers such as C-11 Methionine have a distinct advantage in that the background normal parenchymal uptake is much less thus leading to a higher tumor to background ratio and has shown promising results in various studies (Takenaka et al., 2014; Kim et al., 2010; Deuschl et al., 2018; Minamimoto et al., 2015; Terakawa et al., 2008). In our study, the highest AUC and highest diagnostic accuracy were obtained for $\mathrm{TBR}_{\max }$ with a sensitivity slightly less than that of rCBV ratio ( 81.8 vs. $84.8 \%$ ) and a much higher specificity ( 92.3 vs. $76.9 \%$ ) using a threshold of 1.23. These results are comparable to those of other studies which have reported sensitivity and specificity ranging between $66-91$ and $60-100 \%$ respectively for differentiating recurrent lesion from treatment related changes (Takenaka et al., 2014; Kim et al., 2010; Deuschl et al., 2018; Minamimoto et al., 2015; Terakawa et al., 2008). A metanalysis (Nihashi et al., 2013) revealed pooled sensitivity of $70 \%$ and specificity of $93 \%$ for detection of recurrence in high grade gliomas using C11methionine PET which is comparable to the values obtained in this study. Besides, we also observed that visual analysis of PET images for recurrence showed the highest agreement with the final diagnosis compared to that of MR perfusion and diffusion. This is expected in view of the increased contrast between lesion uptake and brain parenchyma even in lesions located close to the cortex. Our findings are corroborated by another study (Minamimoto et al., 2015) in which no significant difference was seen between visual and quantitative analysis in differentiating recurrent brain lesions from radiation necrosis on C11-methionine PET. However, one of the important disadvantages of C11-methionine is that uptake may also be seen in areas of radiation necrosis (Fig. 7) and acute inflammatory pathology (Fig. 8) leading to a false positive diagnosis 


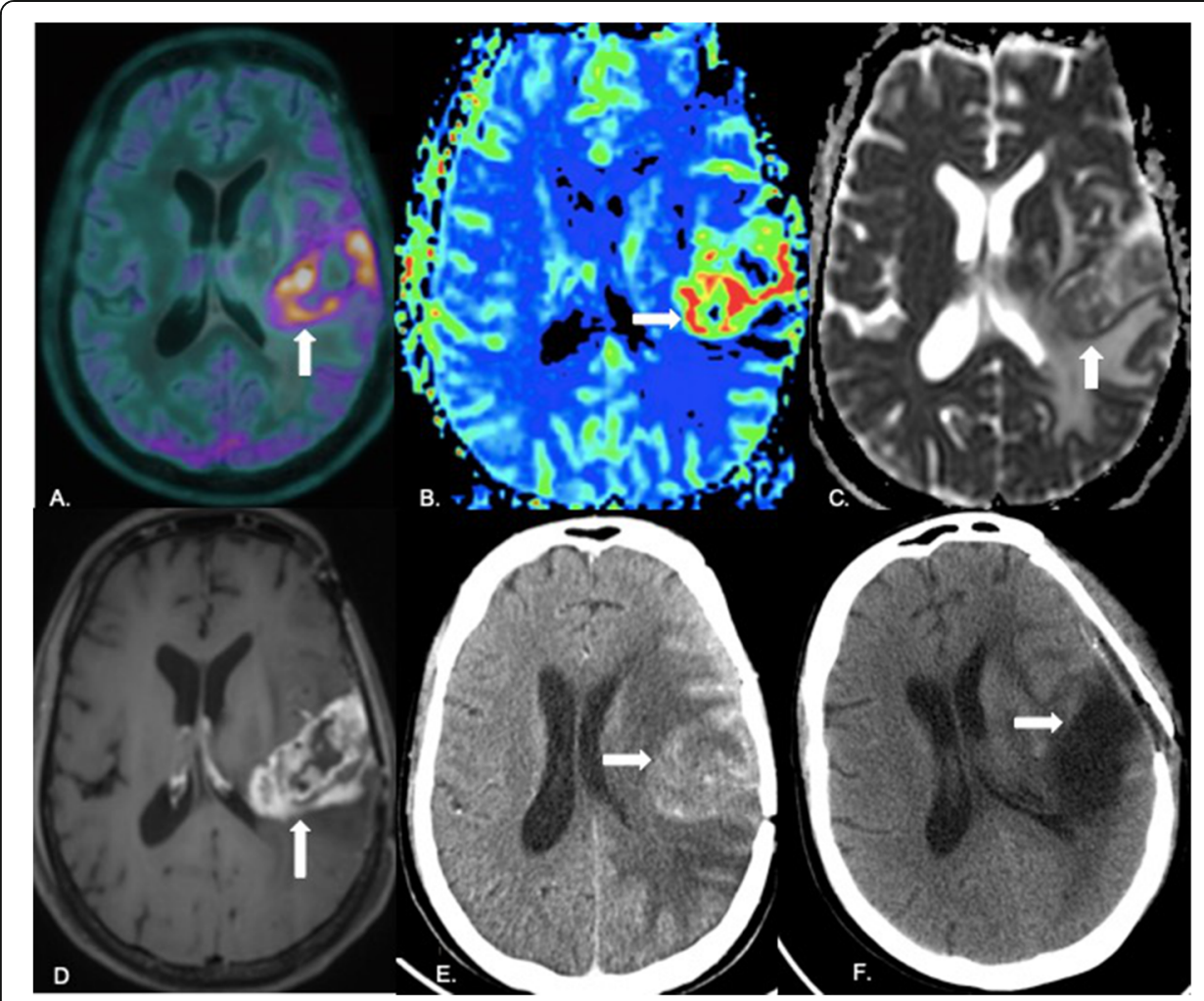

Fig. 5 Concordant PET, perfusion, diffusion and enhancement in a case of recurrence: 40-year-old male diagnosed with anaplastic ODG 15 months back. Underwent surgical excision followed by radiotherapy completed 13 months earlier. PET image overlaid over T1 MPRAGE (A) shows a large area of uptake in the left temporoparietal region (white arrow). rCBV map (B) generated with the leakage correction algorithm shows elevated perfusion in the same region (white arrow) with diffusion restriction on the ADC map (white arrow in $\mathbf{C}$ ). Note enhancement on the post-contrast image (white arrow in $\mathbf{D}$ ). In view of unequivocal evidence of recurrence, surgery was planned. Post-contrast axial $C T$ brain acquired prior to surgery (E) shows an enhancing left parietal lesion (white arrow). Post-operative plain axial CT brain (F) shows the resection cavity (white arrow) with gross total excision. Histopathology revealed recurrent GBM. This is a case of recurrence where the PET, perfusion, diffusion, and contrast images were concordant with good spatial congruence

(Ogawa et al., 1991). In view of uptake by inflammatory cells, it has been reported to show lower diagnostic accuracy compared to other amino acid radiotracers like fluroethyltyrosine (FET) and fluoro-dihydroxyphenylalanine (F-DOPA) (Nihashi et al., 2013; Minamimoto et al., 2015; Galldiks et al., n.d.). In this cohort, we encountered a false positive diagnosis in 4 out of 48 cases $(8.33 \%)$ on methionine PET in which the findings were discordant with MR perfusion and diffusion.

MR perfusion has an established role in the detection of recurrent disease. Perfusion parameters are markers of neovascularization which characterizes progressive disease. DSC-perfusion is the most common method used and has been studied in greater detail compared to other methods like dynamic contrast-enhanced (DCE) perfusion and arterial spin labeling (ASL) (van Dijken et al., 2019). Quantitative assessment of perfusion maps especially rCBV and rCBV ratio calculated in relation to the contralateral normal parenchyma have shown good diagnostic yield in several studies (Barajas Jr et al., 2009; Nael et al., 2018; Wang et al., 2016). We obtained a sensitivity and specificity of 84.8 and $76.9 \%$ using a cut-off value of 1.38 for rCBV ratio to detect recurrence. Our results 


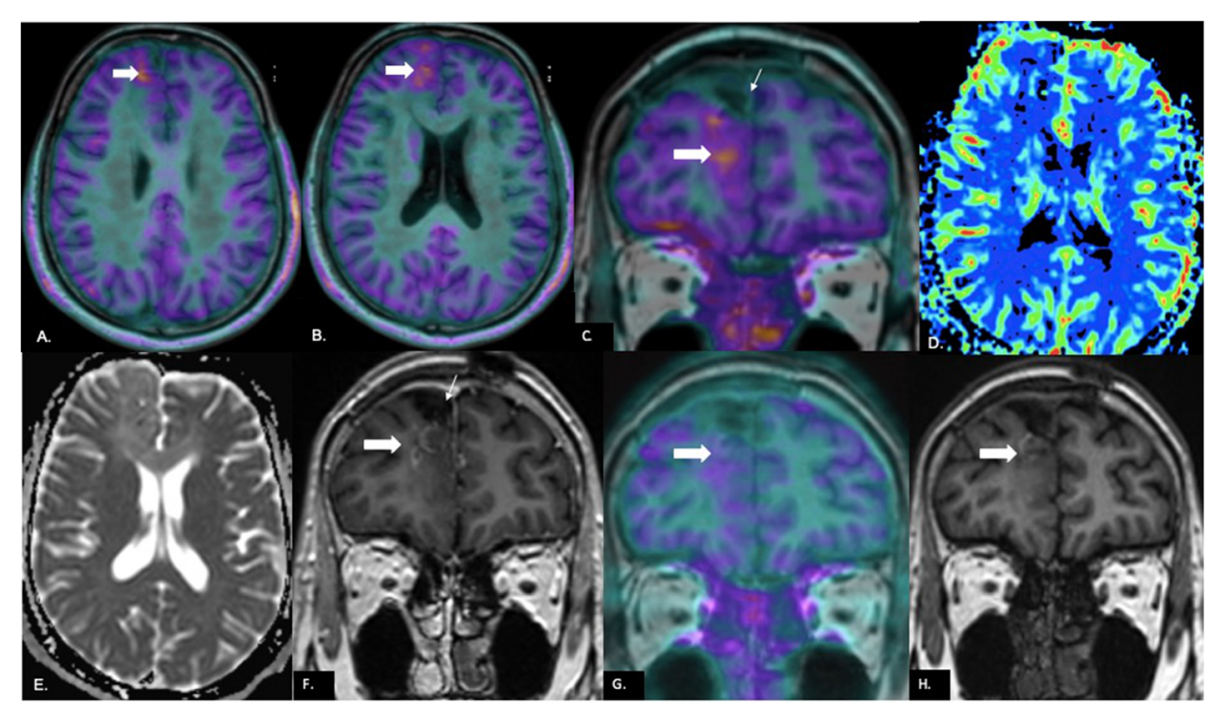

Fig. 6 Discordance between PET, perfusion and diffusion in a case of recurrence: 31-year-old male operated for right frontal ODG 14 months back; last radiation dose 10 months ago. PET images fused with axial T1 MPRAGE (A, B) show an area of uptake in the right superior frontal gyrus (white arrow in A, B). The uptake (block white arrow) is seen along the margins of the resection cavity (small white arrow) on the coronal fused PET-MR image (C). rCBV map (D) generated using the leakage correction algorithm shows no unequivocal elevation of perfusion in the area. There is no diffusion restriction on the ADC map (E). Few foci of enhancement (block white arrow) are seen along the margins of the resection cavity (small white arrow) on post-contrast coronal T1 MPRAGE (F). In view of PET uptake, recurrence was suspected and chemotherapy administered. Follow-up PET image after chemotherapy fused with coronal T1 MPRAGE (G) shows reduction in the degree of uptake (block white arrow) along the resection cavity margin when compared with prechemo coronal PET image in $\mathbf{C}$. Reduction in the degree of enhancement is also seen on the follow-up post-contrast coronal T1 MPRAGE (white arrow in $\mathbf{H}$ ) compared to that in $\mathbf{F}$

are comparable to a pooled analysis which showed sensitivity ranging between 82 and 91\% and specificity between 77 and 91\% for the detection of recurrence using DSC perfusion (van Dijken et al., 2019). However, the threshold rCBV ratio shows wide variation among studies ranging between 0.71 and 3.7 (van Dijken et al., 2019). This may be due to co-existence of recurrent disease and radiation necrosis (Blasel et al., 2016) and leakage across the blood brain barrier leading to errors in estimation since DSC perfusion works on the premise that the contrast agent is confined to the intravascular compartment (van Dijken et al., 2019). We tried to overcome this limitation to some extent by using a leakage correction algorithm to process the perfusion study. Visual assessment of rCBV maps showed only moderate agreement with the final diagnosis which was much less than that seen with amino acid PET. This may be accounted for by the fact that small areas of elevated perfusion or lesions near the cortex are likely to be overlooked in view of the background normally perfused brain parenchyma unlike PET using amino acid tracers where an area of uptake distinctly stands out from the rest of the normal brain. Thus, while diagnostic accuracy of quantitative PET and perfusion parameters are comparable, visual assessment of amino acid PET does score over rCBV maps in detecting recurrence, however, at a cost of false positive diagnosis in some cases.

High cell density and continued cellular proliferation in recurrent tumor restricts the diffusion of water molecules seen as a reduction in the ADC values. However, diffusion imaging is limited by the heterogeneity of lesions leading to a relatively poor diagnostic 


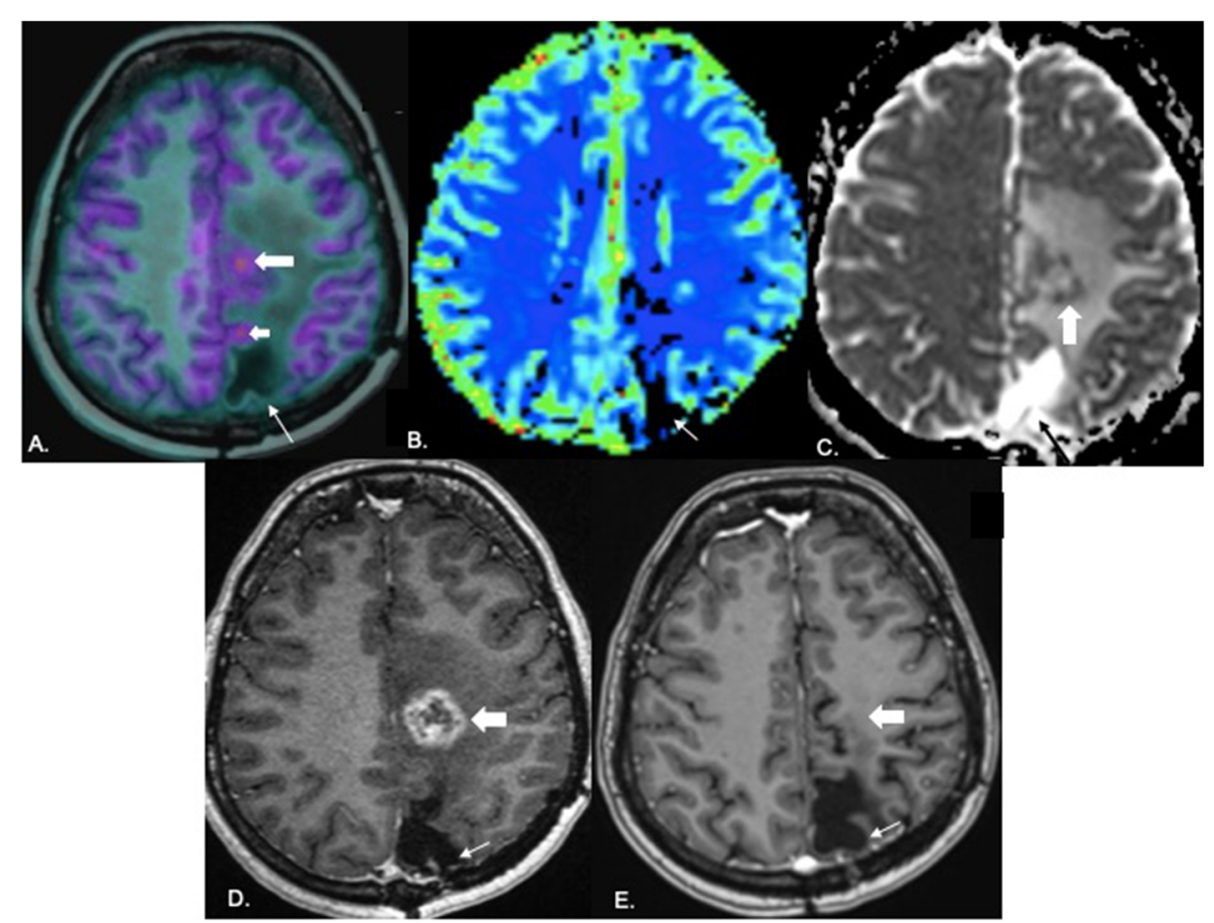

Fig. 7 False positive diagnosis on PET: 34-year-old female diagnosed with anaplastic ODG 2 years back with enhancing lesion on MRI. PET image fused with axial T1 MPRAGE (A) shows an area of uptake along the parasagittal region of the left frontal lobe (large thick arrow) and another area (small thick arrow) along the margin of the resection cavity (linear white arrow) raising a suspicion of recurrence. There is no evidence of elevated perfusion on the rCBV map (B). Resection cavity is shown by white arrow. Peripheral diffusion restriction is seen along the margins of the lesion in the left centrum semiovale (block white arrow in $\mathbf{C}$ ). There is no restriction along the resection cavity (black arrow). Peripheral enhancement is seen along the margins of the lesion in the left centrum semiovale (block white arrow in $\mathbf{D}$ ) with no enhancement along the resection cavity (white arrow). One month follow-up axial post-contrast T1 MPRAGE (E) shows the resection cavity (thin white arrow) with complete resolution of enhancement in the left centrum semiovale (block white arrow) suggestive of treatment related changes excluding recurrence

performance (Soni et al., 2020; Brandes et al., 2008). Among the two quantitative diffusion parameters evaluated in this study, mean ADC and ADC ratio, higher AUC was obtained for ADC ratio with a sensitivity and specificity of 78.1 and $69.2 \%$ respectively for detecting recurrent tumor with threshold of 1.11. Using the visual grading scale, grades higher than grade 1 were sensitive $(84 \%)$ for detecting recurrent disease at the cost of very low specificity of $46.2 \%$. The diagnostic accuracy was $73.65 \%$ comparable with other studies (Jena et al., 2017; Nael et al., 2018). Also, the threshold ADC ratio of 1.11 shows that the ADC in recurrent lesions is nearly the same as that of the normal white matter thus making visual detection of recurrence on diffusion images difficult. Co-existence of areas of necrosis, recurrent tumor, edema and hemorrhage reflects as marked heterogeneity on diffusion images making it difficult to arrive at a specific diagnosis.

Given the different aspects of tumor biology reflected by each of these imaging modalities, it is intuitive that a combination of parameters provides a more comprehensive picture of the lesional and perilesional milieu resulting in better diagnostic performance. This is supported by the findings of our study which showed a significantly higher AUC for a combination of $\mathrm{TBR}_{\max }$ and $\mathrm{rCBV}$ ratio (0.908) compared to rCBV ratio 


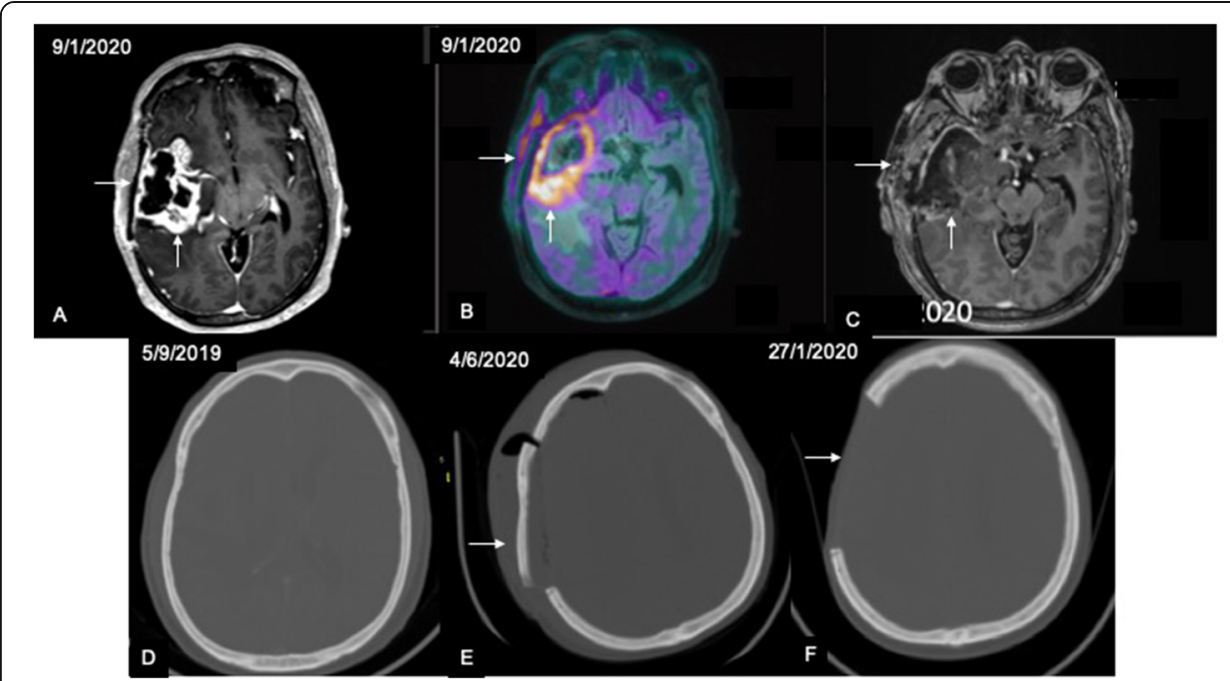

Fig. 8 Sequence of images showing false positive C11 methionine uptake in acute inflammatory pathology: Axial post-contrast T1 MPRAGE (A) in a case of suspected recurrence shows parenchymal enhancement (vertical arrow) suggestive of recurrent lesion with associated enhancement of the overlying bone and soft tissues (horizontal arrow). C11 methionine PET image fused with axial T1 MPRAGE (B) shows tracer uptake along the bone and soft tissues (horizontal arrow) in addition to parenchymal uptake (vertical arrow). Here the uptake in the bone and soft tissues occurred due to osteomyelitis. Follow-up axial post-contrast T1 MPRAGE following chemo/radiotherapy $(\mathbf{C})$ shows reduction in parenchymal enhancement (vertical arrow) with persistent enhancement in bone and soft tissues (horizontal arrow). Sequential axial CT brain images in bone window (D-F) show initial scan (D) with normal bone followed by progressive osteomyelitis in $\mathbf{E}$ and excision of the infected bone in $\mathbf{F}$

alone (0.823) thus improving diagnostic accuracy. The AUC further increased with the addition of ADC ratio (0.913); however, the difference was not statistically significant. Various studies have shown the superiority of combined PET and MRI over either modality alone (Jena et al., 2017; Kim et al., 2010; Ozsunar et al., 2010; Qiao et al., 2019). Adding $\mathrm{TBR}_{\max }$ to choline/creatine ratio (Cho/Cr), rCBV ratio and mean ADC led to an increase in the AUC to 0.932 from 0.913 for MRI alone in a study by Jena et al (Jena et al., 2017). The best diagnostic performance was seen with a combination of $\mathrm{TBR}_{\text {mean }}$, mean $\mathrm{ADC}$, and $\mathrm{Cho} / \mathrm{Cr}(\mathrm{AUC}=.935)$. Our results are concordant with a recent study on C11-Methionine PET with DSC perfusion where they obtained an AUC of 0.953 by combining $\mathrm{TBR}_{\max }$ with $\mathrm{rCBV}$ (Qiao et al., 2019). With the advent of hybrid PET-MRI scanners, combined use of structural and functional MRI with metabolic imaging is emerging as an attractive paradigm for evaluation of post-treatment gliomas. It allows simultaneous or sequential acquisition in a single sitting thus reducing time, overcoming logistic hurdles and improving patient convenience and cooperation. Simultaneous acquisition in the same time frame allows better correlation between the dynamics of various functional parameters like uptake on PET and perfusion on MRI. With combined imaging, there are less chances of a false negative diagnosis. As was seen in our study, in cases with discordance between PET and perfusion and/or diffusion on visual analysis, there was no instance of a false negative diagnosis as all cases with recurrence showed uptake on PET. However, false positive diagnosis of recurrence was made in few cases on PET due to uptake seen in radiation necrosis as well.

Elevated perfusion and lower ADC on MRI showed moderate spatial concordance with PET uptake in 70.9 and $62.5 \%$ cases respectively. A one is to one spatial 
correlation is almost never seen. Discordance rate between DSC perfusion and PET for detection of recurrence in our study was $22.9 \%$ which is comparable to the rates reported in earlier studies (Hatzoglou et al., 2016; Seligman et al., 2019). A hybrid PETMRI study on brain tumors with FET as radiotracer showed poor spatial congruence between PET uptake and elevated perfusion (Filss et al., 2014). Lack of spatial congruence brings to light the fact that areas of increased metabolic uptake on PET and elevated perfusion on MRI represent different aspects of tumor physiology and that elevated perfusion does not directly translate to hypermetabolism and vice versa. The two may co-exist in some regions with variability in rest of the lesion.

Our study had various limitations. It was a retrospective study from a single center. The sample sizes in the groups of recurrence and post-treatment changes without recurrence were discrepant. We did not have histopathology for all the cases as biopsy in cases of treatment related changes raises ethical issues. In addition, surgical decompression may not be considered in all cases of recurrence in view of poor Karnofsky performance score in many patients. Also, tumor segmentation may provide more robust results compared to co-registration alone. In addition, in view of the heterogenous nature of these lesions, ROI analysis alone from a given area without an integrative method like histogram analysis is not representative of the entire lesion.

\section{Conclusion}

Methionine-PET and DSC perfusion are comparable for detecting recurrence in posttreatment gliomas. Diffusion MRI shows lower diagnostic accuracy in view of lesional heterogeneity. Combined PET-MR imaging with C11-methionine as tracer shows superiority over either modality alone and is a feasible option for post-treatment followup of gliomas. PET scores over perfusion as well as diffusion MRI in visually detecting recurrence without quantification. One is to one spatial congruence between the modalities is rarely seen as they reflect different aspects of tumor biology.

Code availability

Standard software applications have been used for data analysis and are mentioned in the methodology.

Consent to participate

Informed consent was obtained from all patients prior to undergoing PET-MR which included consent for participation in research.

\section{Authors' contributions}

The data was analyzed and the first draft prepared by SJ; AA helped with manuscript preparation and investigation. DK was involved in data analysis. CN conceptualized the study, provided data, and revised the manuscript. JS was involved in conceptualization and supervision of the study. PKS was involved in synthesis of the radionuclide used and quality control. NS and AA were involved in the clinical aspects of the study and patient selection. UK was involved in post operative chemoradiotherapy and provided inputs in validating the imaging findings in post treatment glioma patients. VS provide the histopathological diagnosis. All authors read and approved the final manuscript.

\section{Funding}

No funding was received for conducting this study.

\section{Availability of data and materials}

The data used in the study can be provided if required on request from the corresponding author.

\section{Declarations}




\section{Consent for publication}

Informed consent was obtained from all patients prior to undergoing PET-MR which included consent for participation in research and publication.

\section{Competing interests}

The authors declare that they have no competing interest.

\section{Author details}

${ }^{1}$ Sher-i-Kashmir Institute of Medical Sciences, Srinagar, Kashmir 190001, India. ${ }^{2}$ Department of Neuroimaging and Interventional Radiology, National Institute of Mental Health and Neurosciences, Bengaluru, Karnataka 560029, India. ${ }^{3}$ Department of Neurosurgery, National Institute of Mental Health and Neurosciences, Bengaluru, Karnataka 560029, India. ${ }^{4}$ Department of Radiation Oncology, Kidwai Memorial Institute of Oncology, Bengaluru, Karnataka 560029, India. ${ }^{5}$ Department of Neuropathology, National Institute of Mental Health and Neurosciences, Bengaluru, Karnataka 560029, India.

Received: 14 March 2021 Accepted: 21 July 2021

Published online: 18 August 2021

\section{References}

Barajas RF Jr, Chang JS, Segal MR, Parsa AT, McDermott MW, Berger MS, Cha S (2009 Nov) Differentiation of recurrent glioblastoma multiforme from radiation necrosis after external beam radiation therapy with dynamic susceptibilityweighted contrast-enhanced perfusion MR imaging. Radiology. 253(2):486-496. https://doi.org/10.1148/radiol.2532090007

Blasel S, Zagorcic A, Jurcoane A, Bähr O, Wagner M, Harter PN, Hattingen E (2016 Jan) Perfusion MRI in the evaluation of suspected glioblastoma recurrence. Journal of Neuroimaging. 26(1):116-123. https://doi.org/10.1111/jon.12247

Brandes AA, Franceschi E, Tosoni A, Blatt V, Pession A, Tallini G, Bertorelle R, Bartolini S, Calbucci F, Andreoli A, Frezza G (2008 May 1) MGMT promoter methylation status can predict the incidence and outcome of pseudoprogression after concomitant radiochemotherapy in newly diagnosed glioblastoma patients. Journal of Clinical Oncology. 26(13):21922197. https://doi.org/10.1200/JCO.2007.14.8163

Deuschl C, Kirchner J, Poeppel TD, Schaarschmidt B, Kebir S, El Hindy N, Hense J, Quick HH, Glas M, Herrmann K, Umutlu L (2018) 11 C-MET PET/MRI for detection of recurrent glioma. European journal of nuclear medicine and molecular imaging. 45(4):593-601. https://doi.org/10.1007/s00259-017-3916-9

van Dijken BR, van Laar PJ, Smits M, Dankbaar JW, Enting RH, van der Hoorn A (2019 Jan) Perfusion MRI in treatment evaluation of glioblastomas: clinical relevance of current and future techniques. Journal of Magnetic Resonance Imaging. 49(1):11-22. https://doi.org/10.1002/jmri.26306

Filss CP, Galldiks N, Stoffels G, Sabel M, Wittsack HJ, Turowski B, Antoch G, Zhang K, Fink GR, Coenen HH, Shah NJ (2014 Apr 1) Comparison of 18F-FET PET and perfusion-weighted MR imaging: a PET/MR imaging hybrid study in patients with brain tumors. Journal of Nuclear Medicine. 55(4):540-545. https://doi.org/10.2967/jnumed.113.129007

Norbert Galldiks, Philipp Lohmann, Nathalie L Albert, Jörg C Tonn, Karl-Josef Langen, Current status of PET imaging in neurooncology, Neuro-Oncology Advances, Volume 1, Issue 1, May-December 2019,vdz 010.

Hatzoglou V, Yang TJ, Omuro A, Gavrilovic I, Ulaner G, Rubel J, Schneider T, Woo KM, Zhang Z, Peck KK, Beal K (2016 Jun 1) A prospective trial of dynamic contrast-enhanced MRI perfusion and fluorine-18 FDG PET-CT in differentiating brain tumor progression from radiation injury after cranial irradiation. Neuro-oncology. 18(6):873-880. https://doi.org/10.1093/neuonc/nov301

Heiss WD, Raab P, Lanfermann H (2011 Oct 1) Multimodality assessment of brain tumors and tumor recurrence. Journal of Nuclear Medicine. 52(10):1585-1600. https://doi.org/10.2967/jnumed.110.084210

Hustinx R, Pourdehnad M, Kaschten B, Alavi A (2005) PET imaging for differentiating recurrent brain tumor from radiation necrosis. Radiologic Clinics. 43(1):35-47. https://doi.org/10.1016/j.rcl.2004.09.009

Jena A, Taneja S, Jha A, Damesha NK, Negi P, Jadhav GK, Verma SM, Sogani SK (2017) Multiparametric evaluation in differentiating glioma recurrence from treatment-induced necrosis using simultaneous 18F-FDG-PET/MRI: a singleinstitution retrospective study. American Journal of Neuroradiology. 38(5):899-907. https://doi.org/10.3174/ajnr.A5124

Keunen O, Taxt T, Grüner R, Lund-Johansen M, Tonn JC, Pavlin T, Bjerkvig R, Niclou SP, Thorsen F (2014 Sep 30) Multimodal imaging of gliomas in the context of evolving cellular and molecular therapies. Advanced drug delivery reviews. 76:98115. https://doi.org/10.1016/j.addr.2014.07.010

Kim YH, Oh SW, Lim YJ, Park CK, Lee SH, Kang KW, Jung HW, Chang KH (2010 Nov 1) Differentiating radiation necrosis from tumor recurrence in high-grade gliomas: assessing the efficacy of 18F-FDG PET, 11C-methionine PET and perfusion MRI. Clinical neurology and neurosurgery. 112(9):758-765. https://doi.org/10.1016/j.clineuro.2010.06.005

Minamimoto R, Saginoya T, Kondo C, Tomura N, Ito K, Matsuo Y, Matsunaga S, Shuto T, Akabane A, Miyata Y, Sakai S (2015) Differentiation of brain tumor recurrence from post-radiotherapy necrosis with $11 \mathrm{C}$-methionine PET: visual assessment versus quantitative assessment. PloS one. 10(7):e0132515. https://doi.org/10.1371/journal.pone.0132515

Nael K, Bauer AH, Hormigo A, Lemole M, Germano IM, Puig J, Stea B (2018 Jan) Multiparametric MRI for differentiation of radiation necrosis from recurrent tumor in patients with treated glioblastoma. American Journal of Roentgenology. 210(1):18-23. https://doi.org/10.2214/AJR.17.18003

Nihashi T, Dahabreh IJ, Terasawa T (2013 May 1) Diagnostic accuracy of PET for recurrent glioma diagnosis: a meta-analysis, American Journal of Neuroradiology. 34(5):944-950. https://doi.org/10.3174/ajnr.A3324

Nozawa A, Rivandi AH, Kanematsu M, Hoshi H, Piccioni D, Kesari S, Hoh CK (2015 Jun) Glucose-corrected standardized uptake value in the differentiation of high-grade glioma versus post-treatment changes. Nuclear medicine communications. 36(6):573-581. https://doi.org/10.1097/MNM.0000000000000288

Ogawa T, Kanno I, Shishido F, Inugami A, Higano S, Fujita H, Murakami M, Uemura K, Yasui N, Mineura K, Kowada M (1991 May) Clinical value of PET with 18F-fluorodeoxyglucose and L-methyl-11C-methionine for diagnosis of recurrent brain tumor and radiation injury. Acta Radiologica. 32(3):197-202. https://doi.org/10.1177/028418519103200302 
Ostrom QT, Gittleman H, Truitt G, Boscia A, Kruchko C, Barnholtz-Sloan JS (2018 Oct 1) CBTRUS statistical report: primary brain and other central nervous system tumors diagnosed in the United States in 2011-2015. Neuro-oncology 20(suppl_4):iv1i86

Ozsunar Y, Mullins ME, Kwong K, Hochberg FH, Ament C, Schaefer PW, Gonzalez RG, Lev MH (2010 Mar 1) Glioma recurrence versus radiation necrosis?: A pilot comparison of arterial spin-labeled, dynamic susceptibility contrast enhanced MRI, and FDG-PET imaging. Academic radiology. 17(3):282-290. https://doi.org/10.1016/j.acra.2009.10.024

Qiao Z, Zhao X, Wang K, Zhang Y, Fan D, Yu T, Shen H, Chen Q, Ai L (2019 Feb 1) Utility of dynamic susceptibility contrast perfusion-weighted MR imaging and 11C-methionine PET/CT for differentiation of tumor recurrence from radiation injury in patients with high-grade gliomas. American Journal of Neuroradiology. 40(2):253-259. https://doi.org/10.3174/ajnr.A 5952

Ricci PE, Karis JP, Heiserman JE, Fram EK, Bice AN, Drayer BP (1998) Differentiating recurrent tumor from radiation necrosis: time for re-evaluation of positron emission tomography? American Journal of Neuroradiology. 19(3):407-413

Ronca R, Benkheil M, Mitola S, Struyf S, Liekens S (2017 Nov) Tumor angiogenesis revisited: regulators and clinical implications. Medicinal research reviews. 37(6):1231-1274. https://doi.org/10.1002/med.21452

Seeger A, Braun C, Skardelly M, Paulsen F, Schittenhelm J, Ernemann U, Bisdas S (2013 Dec 1) Comparison of three different MR perfusion techniques and MR spectroscopy for multiparametric assessment in distinguishing recurrent high-grade gliomas from stable disease. Academic radiology. 20(12):1557-1565. https://doi.org/10.1016/j.acra.2013.09.003

Seligman L, Kovanlikaya I, Pisapia DJ, Naeger DM, Magge R, Fine HA, Chiang GC (2019 Apr) Integrated PET-MRI for glioma surveillance: perfusion-metabolism discordance rate and association with molecular profiling. American Journal of Roentgenology. 212(4):883-891. https://doi.org/10.2214/AJR.18.20531

Soni N, Ora M, Mohindra N, Menda Y, Bathla G (2020 Sep 1) Diagnostic performance of PET and perfusion-weighted imaging in differentiating tumor recurrence or progression from radiation necrosis in posttreatment gliomas: a review of literature. American Journal of Neuroradiology. 41(9):1550-1557. https://doi.org/10.3174/ajnr.A6685

Stupp R, Mason WP, Van Den Bent MJ, Weller M, Fisher B, Taphoorn MJ, Belanger K, Brandes AA, Marosi C, Bogdahn U, Curschmann J (2005a Mar 10) Radiotherapy plus concomitant and adjuvant temozolomide for glioblastoma. New England journal of medicine. 352(10):987-996. https://doi.org/10.1056/NEJMoa043330

Stupp R, Mason WP, van den Bent MJ, Weller M, Fisher B, Taphoorn MJB, Belanger K, Brandes AA, Marosi C, Bogdahn U, Curschmann J, Janzer RC, Ludwin SK, Gorlia T, Allgeier A, Lacombe D, Cairncross JG, Eisenhauer E, Mirimanoff RO Radiotherapy plus concomitant and adjuvant temozolomide for glioblastoma. N Engl J Med 2005b;352:987-996. [PubMed: 15758009], 10, DOI: https://doi.org/10.1056/NEJMoa043330

Sugahara T, Korogi Y, Tomiguchi S, Shigematsu Y, Ikushima I, Kira T, Liang L, Ushio Y, Takahashi M (2000 May 1) Posttherapeutic intraaxial brain tumor: the value of perfusion-sensitive contrast-enhanced MR imaging for differentiating tumor recurrence from nonneoplastic contrast-enhancing tissue. American Journal of Neuroradiology. 21(5):901-909

Takenaka S, Asano Y, Shinoda J, Nomura Y, Yonezawa S, Miwa K, Yano H, Iwama T (2014) Comparison of 11C-methionine, 11C-choline, and 18F-fluorodeoxyglucose-positron emission tomography for distinguishing glioma recurrence from radiation necrosis. Neurologia medico-chirurgica. 54(4):280-289. https://doi.org/10.2176/nmc.oa2013-0117

Terakawa Y, Tsuyuguchi N, Iwai Y, Yamanaka K, Higashiyama S, Takami T, Ohata K (2008 May 1) Diagnostic accuracy of 11Cmethionine PET for differentiation of recurrent brain tumors from radiation necrosis after radiotherapy. Journal of Nuclear Medicine. 49(5):694-699. https://doi.org/10.2967/jnumed.107.048082

Verma N, Cowperthwaite MC, Burnett MG, Markey MK (2013 May 1) Differentiating tumor recurrence from treatment necrosis: a review of neuro-oncologic imaging strategies. Neuro-oncology. 15(5):515-534. https://doi.org/10.1093/neuonc/nos307

Wang X, Hu X, Xie P, Li W, Li X, Ma L (2015 Jun) Comparison of magnetic resonance spectroscopy and positron emission tomography in detection of tumor recurrence in posttreatment of glioma: a diagnostic meta-analysis. Asia-Pacific Journal of Clinical Oncology. 11(2):97-105. https://doi.org/10.1111/ajco.12202

Wang S, Martinez-Lage M, Sakai Y, Chawla S, Kim SG, Alonso-Basanta M, Lustig RA, Brem S, Mohan S, Wolf RL, Desai A (2016 Jan 1) Differentiating tumor progression from pseudoprogression in patients with glioblastomas using diffusion tensor imaging and dynamic susceptibility contrast MRI. American Journal of Neuroradiology. 37(1):28-36. https://doi.org/10.31 74/ajnr.A4474

Weathers SP, Gilbert MR (2015 Jul 1) Current challenges in designing GBM trials for immunotherapy. Journal of neurooncology. 123(3):331-337. https://doi.org/10.1007/s11060-015-1716-2

Wen PY, Chang SM, Van den Bent MJ, Vogelbaum MA, Macdonald DR, Lee EQ (2017 Jul 20) Response assessment in neurooncology clinical trials. Journal of Clinical Oncology. 35(21):2439-2449. https://doi.org/10.1200/JCO.2017.72.7511

Zikou A, Sioka C, Alexiou GA, Fotopoulos A, Voulgaris S, Argyropoulou MI (2018 Oct) Radiation necrosis, pseudoprogression, pseudoresponse, and tumor recurrence: imaging challenges for the evaluation of treated gliomas. Contrast media \& molecular imaging. 2018:1-6. https://doi.org/10.1155/2018/6828396

\section{Publisher's Note}

